\section{Hier ist das Herz am falschen Fleck}

\author{
Bereits den alten Babyloniern war die \\ Ectopia cordis bekannt, eine seltene \\ Fehlbildung, bei der das Herz durch \\ einen Defekt des Sternums außer- \\ halb des Thorax platziert ist und di- \\ rekt unter der Haut liegt. Die Verän- \\ derung wird häufig bereits pränatal \\ diagnostiziert und hat eine Inzidenz \\ von 5,5-7,9/Mio Geburten. Die Mor- \\ talität liegt mit 50-60\% sehr hoch.
}

- Die Ectopia cordis geht üblicherweise mit weiteren intrathorakalen Anomalien und Defekten einher, so zum Beispiel Defekten der Abdominalwand, des ventralen Zwerchfellanteils und des Perikards. Eine chirurgische Revision ist in der Regel unumgänglich.

In einem ersten Schritt wird das Herz mit Weichteilgewebe überdeckt, später verbringt man es zurück in den knöchernen Thorax. Dabei ist vor allem ein Abknicken der großen Gefäße zu vermeiden.

Die hier abgebildete Patientin hatte zusätzlich zur Ectopia cordis auch eine Fallotsche Tetralogie, eine Pulmonal-
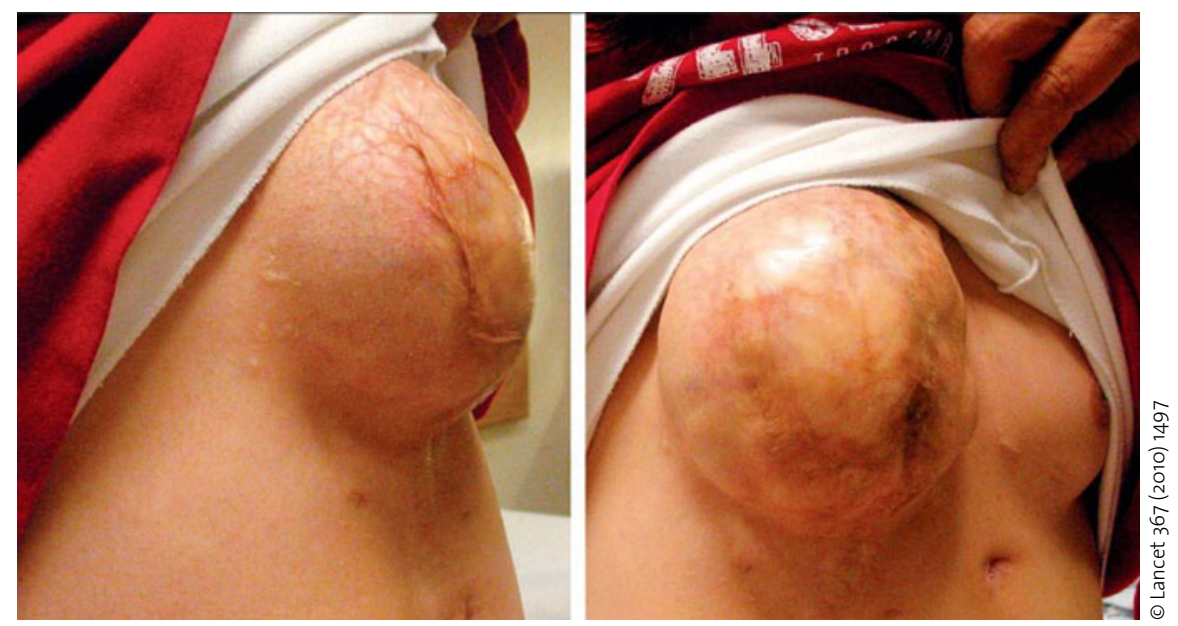

Ectopia cordis: Das Herz befindet sich vollständig außerhalb der knöchernen Brustwand und wird nur von Haut bedeckt

atresie und einen rechten Aortenbogen mit aberrierender linker A. subclavia. Auch diese Anomalien wurden korrigiert. Nachdem im vorliegenden Fall aber die Leber einen großen Teil des Brustraums einnahm, konnte das Herz nicht intrathorakal zurückverlagert werden.

Das hier dargestellte Mädchen ist bereits 17 Jahre alt und stellt somit den am längsten überlebenden Fall einer Ectopia cordis dar. Zum Schutz des Herzens vor Traumata verwendet sie eine Art von Korsett.
Leider wird nicht mitgeteilt, wie die psychische Entwicklung des Kindes verlaufen ist. Es würde auch Herzchirurgen gut anstehen, wenn sie auch diesen Aspekt des Lebens im Auge behielten.

H. S. FüEßL =

\title{
Grippewelle in öffentlichen Verkehrsmitteln
}

\begin{abstract}
Dass die Ansteckungsgefahr während der Grippesaison in öffentlichen Verkehrsmitteln besonders hoch ist, ist eine Binsenweisheit. Wissenschaftlich näher untersucht wurde die Beziehungen zwischen öffentlichem Transport und akuten respiratorischen Infektionen aber bisher nicht.
\end{abstract}

— Forscher der University of Nottingham (England) führten ihre Fall-Kontroll-Studie während der Grippesaison 2008/2009 durch. In die Studie aufgenommen wurden 138 Patienten, die einen Allgemeinmediziner konsultierten, 72 wegen akuter Atemwegsinfektionen und 66 Patienten mit anderen Erkran- kungen. Die Probanden füllten einen Fragebogen zur Nutzung des öffentlichen Nahverkehrs fünf Tage vor dem Auftreten der Symptome aus. Multiple logistische Regressionsmodelle wurden zur Auswertung eingesetzt.

Für Gelegenheitsfahrer war das Risiko am größten. Passagiere von Bus und Tram mussten sechsmal häufiger $(\mathrm{OR}=$ 5,94; 95\%-KI: 1,33-26,5) mit einer akuten Atemwegserkrankung zum Arzt. Das Risiko scheint in Abhängigkeit von der Zahl der Fahrten pro Woche modifiziert zu sein, es ist aber nicht statistisch signifikant: 1-3 Fahrten pro Woche: $\mathrm{OR}=0,54 ; 95 \%-\mathrm{KI}: 0,15-1,95$; $>3$ Fahrten pro Woche: $\mathrm{OR}=0,37$; 95\%-KI: 0,13-1,06.

\section{Kommentar}

Die Studie ist sehr klein. Größere Untersuchungen müssen folgen. Aber die Autoren meinen, dass die Resultate auf jeden Fall den Rat rechtfertigen, in öffentlichen Verkehrsmitteln auf die Hygiene zu achten, besonders in den Wintermonaten während der Grippesaison und wenn man selbst erkrankt ist und Infektionsquelle für den Mitfahrer sein kann. K. MALBERG -

\section{- J. Troko et al.}

(J.N. Van Tam, Div Epidemiol. \& Public Health, Univ. of Nottingham, Clin Sci Bldg., City Hospital, Nottingham, NG 5 1PB; e-mail: jvt@ nottingham.ac.uk ) Is public transport a risk factor for acute respiratory infection? Published online 14 January 2011; BMC Infectious Diseases 2011; 11:16; doi: 1186/1471-2334-11-16 